\title{
ALTERAÇÃO FLORÍSTICA DE ÁREAS DE FLORESTAS EXPLORADAS CONVENCIONALMENTE EM PLANOS DE MANEJO, NOS DOMÍNIOS DE FLORESTA ATLÂNTICA, MINAS GERAIS-BRASIL ${ }^{1}$
}

\author{
Danilo José da Silva Coelho² e Agostinho Lopes de Souza ${ }^{3}$
}

\begin{abstract}
RESUMO - Este estudo, realizado em fragmentos de Florestas Estacionais Semideciduais na Zona da Mata de Minas Gerais, teve como objetivo analisar as alterações na composição de espécies em área de manejo florestal, tendo como testemunha a área de reserva legal. Decorrido o tempo de exploração florestal de cada PMF e comparando as áreas de manejo florestal e áreas de reserva legal, verificou-se que, com relação a alterações na diversidade das espécies arbóreas dos $\mathrm{PMF}_{\mathrm{S}}$, os verificadores grupo ecológico, grupo de uso e grupo de espécies raras indicaram que as áreas de manejo florestal de todos os $\mathrm{PMF}_{\mathrm{S}}$ são iguais estatisticamente $(\mathrm{P}>0,05)$ às áreas de reserva legal. O verificador riqueza de espécies evidenciou que as áreas de manejo florestal e as áreas de reserva legal dos $\mathrm{PMF}_{28}$ e $\mathrm{PMF}_{29}$ são estatisticamente iguais $(\mathrm{P}>0,05)$, ao passo que nos $\mathrm{PMF}_{30}$ e $\mathrm{PMF}_{16}$ são estatisticamente diferentes $(\mathrm{P} \leq 0,05)$. $\mathrm{O}$ verificador diversidade de espécies indicou que as áreas de manejo florestal são estatisticamente diferentes $(\mathrm{P}>0,05)$ das áreas de reserva legal nos $\mathrm{PMF}_{29}, \mathrm{PMF}_{30}$ e $\mathrm{PMF}_{16} \mathrm{e}$ estatisticamente iguais $(\mathrm{P} \leq 0,05)$ no $\mathrm{PMF}_{28}$. A maior similaridade de espécies ocorreu entre os agrupamentos formados pelos $\mathrm{PMF}_{29 \mathrm{AMF}}$ e $\mathrm{PMF}_{29 \mathrm{ARL}}$ e a menor, entre os agrupamentos formados pelos $\mathrm{PMF}_{29 \mathrm{ARL}}, \mathrm{PMF}_{29 \mathrm{AMF}}$, $\mathrm{PMF}_{28 \mathrm{ARL}}$ e $\mathrm{PMF}_{16 \mathrm{ARL}}, \mathrm{PMF}_{16 \mathrm{AMF}}, \mathrm{PMF}_{30 \mathrm{ARL}}, \mathrm{PMF}_{30 \mathrm{AMF}}$ e $\mathrm{PMF}_{28 \mathrm{AMF}}$.
\end{abstract}

Palavras-chave: Floresta Estacional Semidecidual, composição florística, fitossociologia e manejo florestal.

\section{FLORISTIC CHANGES IN CONVENTIONALLY EXPLOITED FOREST AREAS IN MANAGEMENT PLANS, IN THE ATLANTIC FOREST DOMAIN, MINAS GERAIS STATE-BRAZIL}

\begin{abstract}
The objective of the present study was to analyze the changes in the composition of species, in forest management areas having as control the legal reserve area, and it was carried out in fragments of semideciduous seasonal forests in the Zona da Mata Region of Minas Gerais State. Following the harvest of each FMP, the comparison between forest management areas and legal reserve areas showed that, in relation to changes in diversity of the FMP tree species, the verifiers ecological group, use group and rare species group indicated that the forest management areas of all the FMPs were statistically equal $(P>0.05)$ to the legal reserve areas. The verifier species richness indicated that the forest management areas and the legal reserve areas of the $F M P_{28}$ and $F M P_{29}$ were statistically equal $(P>0.05)$, whereas the $F M P_{30}$ and $F M P_{16}$ were statistically different $(P £ 0.05)$. The verifier species diversity indicated that the forest management areas were statistically different $(P>0.05)$ from the legal reserve areas in $F M P_{29}, F M P_{30}$ and $F M P_{16}$ and statistically equal ( $P £ 0.05)$ in the $F M P_{28}$. The greatest similarity of species occurred between the groups formed by the $F M P_{29 A M F}$ and $F M P_{29 A R L}$, and the smallest similarity occurred between the groups formed by $F M P_{29 A R L}, F M P_{29 A M F}$, $F M P_{28 A R L}$, and $F M P_{16 A R L}, F M P_{16 A M F}, F M P_{30 A R L}, F M P_{30 A M F}, F M P_{28 A M F}$.
\end{abstract}

Keywords: Semideciduous Seasonal Forest, floristic composition, phytosociology and forest management.

\footnotetext{
${ }^{1}$ Recebido em 26.04.2006 e aceito para publicação em 16.12.2006.

${ }^{2}$ Instituto Estadual de Florestas - MG. E-mail: <djcoelho@ufv.br>.

${ }^{3}$ Departamento de Engenharia Florestal da Universidade Federal de Viçosa. E-mail: <alsouza@ufv.br>.
} 


\section{INTRODUÇÃO}

Em Minas Gerais, a composição florística de comunidades florestais exploradas convencionalmente em planos de manejo, nos domínios de floresta atlântica, ainda é pouco conhecida.

As áreas estudadas servem para gerar conhecimentos necessários ao aprimoramento das técnicas de manejo florestal, com a conseqüente melhora na conservação e proteção da flora e dos recursos associados, como solo, água e fauna.

De acordo com Jardim et al. (1993), a atividade de exploração florestal infuencia consideravelmente a diversidade de espécies e, conseqüentemente, a composição florística do local e que também sofre influência do tamanho das clareiras (WHITMORE, 1978, 1989; DENSLOW, 1980, 1987) e do tipo de exploração, se convencional ou planejada (VIDAL et al., 1998).

O Instituto Estadual de Florestas de Minas Gerais, órgão responsável pela gestão dos recursos florestais do Estado, protocolou planos de manejo para exploração florestal em várias regiões do Estado. Nas áreas de formações florestais foram protocolados, nos escritórios do IEF-MG, 132 planos no período de 1993 a 1998, sendo $70(53,07 \%)$ na microrregião da Zona da Mata, $60(45,46 \%)$ na microrregião Centro-Sul e $2(1,31 \%)$ na microrregião Nordeste. Desse total, 78 planos $(58,2 \%)$ possuem áreas de exploração de 10 hectares, $33(24,62 \%)$ entre $10 \mathrm{e}$ 20 hectares e $22(16,42 \%)$ acima de 50 hectares, caracterizando o manejo em pequenos fragmentos florestais, o que dificulta, sobremaneira, a sua fiscalização e o seu monitoramento (IEF, 2000)

Nesse contexto, este trabalho teve por objetivo de avaliar, por meio de indicadores e verificadores, as alterações na composição de espécies de planos de manejo florestal $\left(\mathrm{PMF}_{\mathrm{s}}\right)$ previamente selecionados e representativos da população dos $\mathrm{PMF}_{\mathrm{S}}$ autorizados no Estado.

\section{MATERIAL E MÉTODOS}

\section{1. Área de estudo}

O estudo foi realizado nas fazendas Amoras $\left(\mathrm{PMF}_{28}\right)$, Maia-Vassorouras $\left(\mathrm{PMF}_{29}\right)$ e Retiro $\left(\mathrm{PMF}_{30}\right)$, nos Municípios de Porto Firme, e fazenda Alves ( $\left.\mathrm{PMF}_{16}\right)$, no Município de Rio Espera, localizados na mesorregião Zona da Mata, no Sudeste de Minas Gerais, entre as coordenadas geográficas $19^{\circ} 45^{\prime}$ e $22^{\circ} 15^{\prime} \mathrm{S}$ e $41^{\circ} 15^{\prime}$ e $44^{\circ} 15^{\prime} \mathrm{W}$.

A região apresenta relevo fortemente ondulado e montanhoso, com encostas de perfil convexo-côncavo em vales de fundo chato, formados por terraços e leitos maiores, onde ocorrem cursos d'água. A temperatura média anual oscila em torno de $18,5^{\circ} \mathrm{C}$, e o índice médio pluviométrico anual é de aproximadamente $1.380 \mathrm{~mm}$ (ALMG, 2005).

As formações florestais incluem-se no domínio da floresta Atlântica (floresta estacional semidecidual) (VELOSO, 1992). Seus agrupamentos remanescentes mais expressivos localizam-se nas encostas superiores dos terrenos da região. Ainda que variada quanto à estrutura e composição, destacam-se quanto à flora Copaifera langsdorffii Desf. (copaíba), Ocotea sp. e Nectandra sp. (Canelas), Schizolobium parahyba (Vell.) Blake (guapuruvu), Cedrela fissilis Vell. (cedro), Plathymenia foliolosa Benth. (vinhático), Aspidosperma polyneuron Müll. Arg. (peroba-rosa) e Cariniana legalis Kuntze (jequitibá-rosa). Outras extensões da floresta estacional semidecidual na região são as galerias ao longo dos cursos d'água (floresta de galeria, mata ciliar), ocupando áreas em condições de umidade permanente (IEF, 1994).

\subsection{Amostragem}

Foram estabelecidas e medidas 20 parcelas de área fixa de $500 \mathrm{~m}^{2}(10 \times 50 \mathrm{~m})$, por PMF, sendo 10 parcelas em área de manejo florestal e 10 em área de reserva legal. As parcelas foram alocadas de forma sistemática, de modo a contemplar os diferentes gradientes de declividade ao longo do terreno (Figura 1). Todos os indivíduos vivos ou mortos em pé, com CAP (circunferência a 1,30 $\mathrm{m}$ do solo) igual ou superior a $15 \mathrm{~cm}$, foram medidos e as alturas total e comercial, estimadas, coletando-se o material botânico para identificação. A identificação taxonômica do material botânico, em níveis de família, gênero e espécies, foi realizada com o auxílio de literatura especializada (LORENZI,1992), consulta a herbário e, quando necessário, a especialista. Todas as espécies tiveram suas sinonímias verificadas pela literatura mais recente.

As espécies amostradas em grupos ecológicos e grupos de usos foram classificadas com o auxílio da literatura especializada e de observações de campo sobre o comportamento delas. 


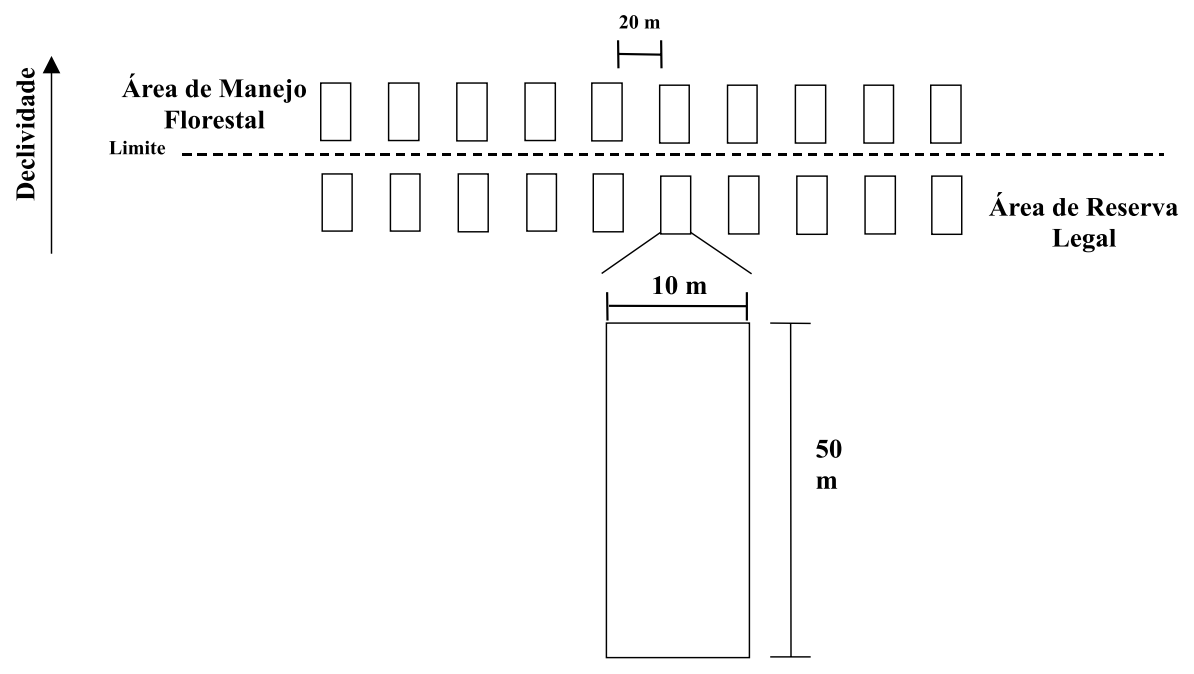

Vegetação arbórea: dap $\geq 5 \mathrm{~cm}$

Figura 1 - Desenho do sistema de amostragem executado nos planos de manejo florestal selecionados com base na análise de agrupamento, para serem inventariados no campo.

Figure 1 -Diagram of the sampling system performed in the selected forest management plans based on the cluster analysis, to be surveyed in the field.

\subsection{Análise dos dados}

Para realizar a análise estatística, foram admitidos dois tratamentos (área de manejo florestal e área de reserva legal), com 10 repetições cada. Para as variáveis em estudo, foram aplicados testes de normalidade de Lillierfors (LILLIEFORS, 1967) e os de homogeneidade das variâncias de Cochran e Barllet (WINER, 1962).

Os resultados a partir dos levantamentos florísticos e estruturais das áreas de manejo florestal e áreas de reserva legal foram submetidos ao teste t, de Student, a 5\% de probabilidade, para verificar a existência de alterações significativas entre as médias dos verificadores analisados.

Para comparar índices de Shannon entre as áreas de manejo florestal e de reserva legal, foi realizado o teste t, de Student (HUTCHESON, 1970; MAGURRAN, 1988).

O intervalo de confiança do índice de Shannon foi calculado pelo método de Jackknife, segundo Neter et al. (1992) e Heltshe e Forrester (1983).

Os dados de campo das parcelas foram processados utilizando o programa MATA NATIVA 2.0 (CIENTEC, 2006), sendo as análises estatísticas executadas pelo software SAEG (2005).

\section{RESULTADOS E DISCUSSÃO}

\subsection{Riqueza florística}

Foram amostradas no $\mathrm{PMF}_{28} 83$ espécies, distribuídas em 33 famílias botânicas (Quadro 1). As famílias com maior número de espécies foram Leg. Mimosoideae, com sete espécies (8,4\%); Annonaceae e Leg. Papilionoideae, com seis espécies cada $(7,2 \%)$; e Euphorbiaceae, com quatro espécies $(4,8 \%)$, perfazendo um total de $27,7 \%$ das espécies relacionadas. $\mathrm{Na}$ área de reserva legal, verificaram-se 66 espécies, distribuídas em 34 famílias botânicas. As famílias com maior número de espécies foram Annonaceae, com seis espécies $(9,1 \%)$; Leg. Papilionoideae, com seis espécies $(9,1 \%)$; Leg. Caesalpinioideae, com cinco espécies (7,6\%); e Euphorbiaceae, com quatro espécies $(6,1 \%)$, perfazendo um total de $31,8 \%$ da espécies relacionadas (Figura 2AB).

No $\mathrm{PMF}_{29}$, oito anos após as atividades de exploração florestal foram amostradas, na área de manejo florestal, 46 espécies, distribuídas em 25 famílias botânicas. As famílias com maior número de representantes taxonômicos foram Annonaceae, com seis espécies (13\%); e Flacourtiaceae, Leg. Caesalpinioideae e Leg. Mimosoideae, com três espécies $(6,5 \%)$, perfazendo 
um total de 32,6\% das espécies. Na área de reserva legal, verificou-se 59 espécies distribuídas em 29 famílias botânicas. As famílias com maior número de representantes taxonômicos foram Annonaceae, com seis espécies (10,2\%); e Euphorbiaceae, Lauraceae e Leg. Mimosoideae, com cinco espécies $(8,5 \%)$, perfazendo um total de $35,6 \%$ das espécies (Figura 2CD).

No $\mathrm{PMF}_{30}$, nove anos após as atividades de exploração florestal foram relacionadas, na área de manejo florestal, 68 espécies, distribuídas em 32 famílias botânicas. As famílias com maior número de espécies foram: Leg. Caesalpinioideae, com sete espécies (10,3\%); Leg. Mimosoideae, com seis espécies (8,8\%); Annonaceae, com cinco espécies (7,3\%); e Euphorbiaceae, com quatro espécies $(5,9 \%)$, perfazendo um total de $32,3 \%$ das espécies. Na área de reserva legal, verificaram-se 75 espécies, distribuídas em 31 famílias botânicas. As famílias com maior número de espécies foram Annonaceae e Leg. Mimosoideae, com sete espécies $(9,3 \%)$ cada; Flacourtiaceae, com cinco espécies $(6,7 \%)$; e Euphorbiaceae, com quatro espécies $(5,3 \%)$, perfazendo um total de 30,7\% das espécies (Figura 2EF). No PMF , $_{16}$, 11 anos após as atividades de exploração florestal foram relacionados, na área de manejo florestal, 109 espécies, distribuídas em 40 famílias botânicas. As famílias com maior número de espécies foram: Leg. Caesalpinioideae, com nove espécies $(8,2 \%)$; Euphorbiaceae, com oito espécies (7,3\%); e Annonaceae e Lauraceae com sete espécies $(6,4 \%)$ cada; perfazendo um total de $28,4 \%$ das espécies. Na área de reserva legal, verificaramse 96 espécies, distribuídas em 35 famílias botânicas. As famílias com maior número de representantes taxonômico foram: Euphorbiaceae, com oito indivíduos (8,3\%); Annonaceae e Leg. Caesalpinioideae, com
7 espécies $(7,3 \%)$ cada; e Flacourtiaceae, com seis espécies $(6,2 \%)$, perfazendo um total de $29,2 \%$ das espécies (Figura 2GH).

Utilizando a riqueza de espécies, como verificador de mudanças da composição de espécies em áreas de manejo florestal e áreas de reserva legal, observouse maior riqueza nas áreas de manejo florestal nos $\mathrm{PMF}_{28}$ e PMF ${ }_{16}$ Isso decorre, provavelmente, das atividades de exploração florestal, que resultam na abertura de clareiras, criando, assim, novas ecounidades (GOMES, 2004), que vêm sendo ocupadas por espécies de diferentes categorias sucessionais ou grupos ecológicos. Esse comportamento foi descrito por Whitmore (1987), Denslow (1980), Whitmore (1989) e Denslow (1987) e constatado por Jardim et al. (1993) em floresta amazônica.

Nos $\mathrm{PMF}_{29}$ e $\mathrm{PMF}_{30}$, decorridos, respectivamente, oito e nove anos da execução das atividade de exploração, houve diferença significativa $(\mathrm{P} \leq 0,05)$ entre o número de espécies amostradas, indicando que a riqueza de espécies das áreas de manejo florestal ainda é inferior à riqueza de espécies das áreas de reserva legal.

No $\mathrm{PMF}_{16}$, decorridos 11 anos das atividades de exploração florestal, não houve diferença significativa ( $\mathrm{P}>0,05)$ no número de espécies amostradas na área de manejo florestal em relação à área de reserva legal, embora seja maior a riqueza na área de manejo florestal.

No $\mathrm{PMF}_{29}$, decorridos 10 anos da exploração florestal, embora apresente maior riqueza na área de reserva legal, verificou-se que não houve diferença significativa $(\mathrm{P}>0,05)$ entre o número de espécies amostrada na área de manejo florestal e o da área de reserva legal, indicando que a área de manejo florestal manteve a riqueza de espécies.

Quadro 1 - Números de árvores por hectare, famílias, espécies e espécies raras, em áreas de manejo florestal (AMF) e áreas de reserva legal (ARL) respectivamente, dos $\mathrm{PMF}_{28}, \mathrm{PMF}_{29}, \mathrm{PMF}_{30}$ e $\mathrm{PMF}_{16}$, nos Municípios de Porto Firme e Rio Espera, Minas Gerais, em 2005

Table 1 - Number of trees per hectare, families, species and rare species, respectively, in forest management areas (AMF) and legal reserve areas (ARL), of the $F M P_{28}, F M P_{29}, F M P_{30}$ and $F M P_{16}$, in the municipalities of Porto Firme and Rio Espera, Minas Gerais State, in 2005

\begin{tabular}{|c|c|c|c|c|c|c|c|c|}
\hline \multirow[t]{3}{*}{ Parâmetro } & \multicolumn{8}{|c|}{ Condições de Floresta } \\
\hline & \multicolumn{4}{|c|}{ AMF } & \multicolumn{4}{|c|}{ ARL } \\
\hline & $\mathrm{PMF}_{28}$ & $\mathrm{PMF}_{29}$ & $\mathrm{PMF}_{30}$ & $\mathrm{PMF}_{16}$ & $\mathrm{PMF}_{28}$ & $\mathrm{PMF}_{29}$ & $\mathrm{PMF}_{30}$ & $\mathrm{PMF}_{16}$ \\
\hline $\mathrm{N}^{\circ}$ de árvores por hectare & 1.500 & 1.880 & 1.488 & 2.162 & 1.468 & 1.946 & 1.524 & 1.508 \\
\hline $\mathrm{N}^{\circ}$ de Famílias & 33 & 25 & 32 & 40 & 34 & 29 & 31 & 35 \\
\hline $\mathrm{N}^{\circ}$ de espécies & 83 & 46 & 68 & 109 & 66 & 59 & 75 & 96 \\
\hline $\mathrm{N}^{\circ}$ de espécies raras & 17 & 13 & 22 & 25 & 17 & 11 & 24 & 21 \\
\hline
\end{tabular}

R. Árvore, Viçosa-MG, v.31, n.2, p.247-256, 2007 


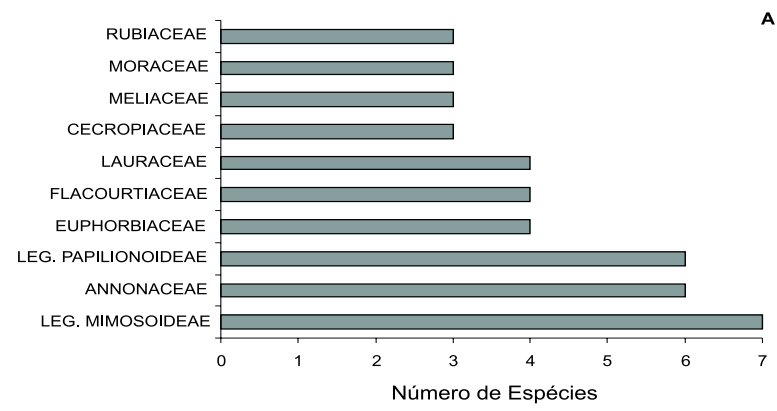

A
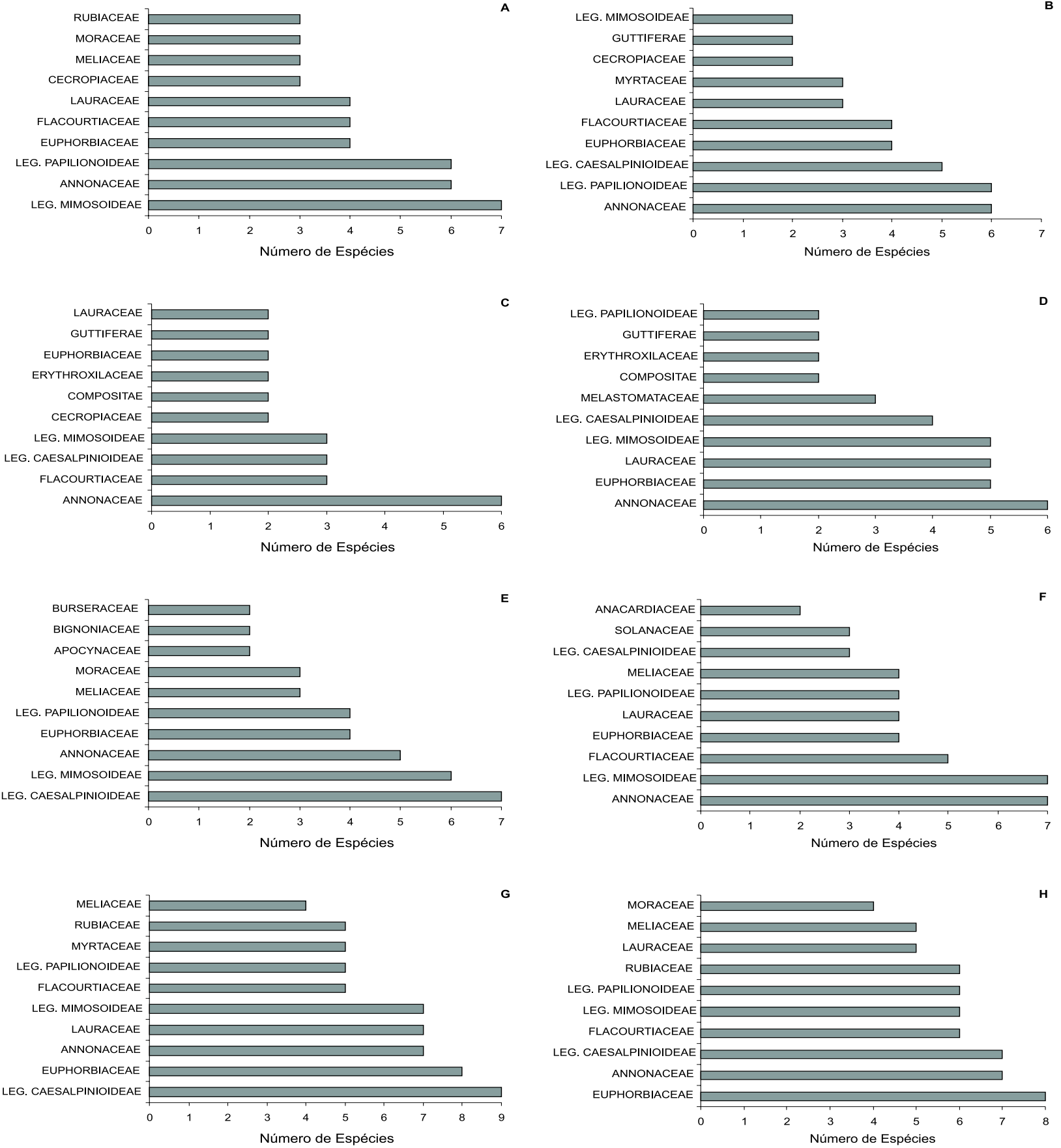

Figura 2 - Número de espécies das famílias de maior valor de importância, amostradas nos $\mathrm{PMF}_{28}(\mathrm{~A}=\mathrm{AMF}, \mathrm{B}=\mathrm{ARL}$ ), $\mathrm{PMF}_{29}(\mathrm{C}=\mathrm{AMF}, \mathrm{D}=\mathrm{ARL}), \mathrm{PMF}_{30}(\mathrm{E}=\mathrm{AMF}, \mathrm{F}=\mathrm{ARL})$ e $\mathrm{PMF}_{16}(\mathrm{G}=\mathrm{AMF}, \mathrm{H}=\mathrm{ARL})$, nos Municípios de Porto Firme e Rio Espera, Minas Gerais, em 2005.

Figure 2 -Number of species of the families with greater importance value sampled in the $F M P_{28}(A=A M F, B=A R L)$, $F M P_{29}(C=A M F, D=A R L), F M P_{30}(E=A M F, F=A R L)$ and $F M P_{16}(G=A M F, H=A R L)$, municipalities of Porto Firme and Rio Espera, Minas Gerais State, in 2005. 


\subsection{Grupo ecológico}

Os resultados apresentados na Tabela 2 indicam que em todos os $\mathrm{PMF}_{\mathrm{s}}$ predominaram os indivíduos de espécies secundárias iniciais em áreas de manejo florestal e áreas de reserva legal. Pode-se inferir, primeiro, que se trata de florestas secundárias em estágio médio de sucessão e, segundo, que até mesmo as áreas de reserva legal teriam sofrido explorações de madeira não-autorizadas e, por fim, que as áreas já tinham histórico de exploração florestal anteriormente à implantação dos $\mathrm{PMF}_{\mathrm{s}}$.

Nos $\mathrm{PMF}_{30}$ e $\mathrm{PMF}_{16}$, os indivíduos de espécies pioneiras predominaram em relação às espécies secundárias iniciais e tardias, tanto nas áreas de manejo florestal quanto nas de reserva legal. Então, pode-se inferir que as condições ambientais nas áreas de manejo florestal e naquelas de reserva legal favoreceram o estabelecimento dos indivíduos de espécies pioneiras.

O número de indivíduos de espécies secundárias tardias maior nas áreas de manejo florestal, em relação às áreas de reserva legal, pode ser devido ao processo de exploração seletiva, com a retirada apenas de indivíduos com dap menor, favorecendo as espécies secundárias tardias.

Contudo, o verificador grupoecológico indicou diferenças não-significativas $(\mathrm{P}>0,05)$ entre as áreas de manejo florestal e as de reserva legal, decorridos 10, 8, 9, e 11 anos da execução das atividades de exploração florestal, respectivamente nos $\mathrm{PMF}_{28}, \mathrm{PMF}_{29}, \mathrm{PMF}_{30}$ e $\mathrm{PMF}_{16}$.

\subsection{Grupo de uso}

Decorridos $10\left(\mathrm{PMF}_{28}\right), 8\left(\mathrm{PMF}_{29}\right), 9\left(\mathrm{PMF}_{30}\right) \mathrm{e}$ 11 anos $\left(\mathrm{PMF}_{16}\right)$, observou-se (Tabela 3$)$ um predomínio das espécies do grupo de uso 1 (energia) em relação às espécies do grupo de uso 2 (serraria), nos $\mathrm{PMF}_{28}$ e $\mathrm{PMF}_{29}$ (área de manejo florestal) e $\mathrm{PMF}_{28}, \mathrm{PMF}_{30} \mathrm{e}$
$\mathrm{PMF}_{16}$ (área de reserva legal). $\mathrm{No} \mathrm{PMF}_{29}$ (área de reserva legal) predominaram as espécies do grupo de uso 2 (serraria).

O verificador grupo de uso indicou diferenças nãosignificativas $(\mathrm{P}>0,05)$ entre as áreas de manejo florestal e as de reserva legal, de todos os $\mathrm{PMF}_{\mathrm{s}}$.

\subsection{Espécies raras}

Consideraram-se espécies raras ou de baixa densidade (Quadro 1) aquelas que apresentaram até dois indivíduos por hectare para o nível de inclusão (DAP $>5,0 \mathrm{~cm})$. No $\mathrm{PMF}_{28}$, na área de manejo florestal, das 83 espécies amostradas, 17 (20,48\%) foram consideradas raras e na área de reserva legal, das 66 espécies amostradas, 17 (25,76\%) foram raras. As espécies raras comuns às duas áreas foram Hortia arborea Engl. e Machaerium nyctitans (Vell.) Benth. No $\mathrm{PMF}_{29}$, na área de manejo florestal, do total de 46 espécies amostradas, 13 espécies $(28,56 \%)$ foram consideradas raras e na área de reserva legal, dentre as 59 espécies amostradas, $11(18,64 \%)$ foram raras. As espécies raras, comuns às duas áreas foram: Dalbergia nigra Allemão ex Benth., Inga sp. e Vernonia sp. No $\mathrm{PMF}_{30}$, na área de manejo florestal, das 66 espécies amostradas, $22(32,35 \%)$ foram consideradas raras e na área de reserva legal, das 75 espécies amostradas, 24 (32,0\%) foram raras. As espécies raras comuns às duas áreas foram Cordia sellowiana Cham., Jacaranda puberula Cham. e Luehea grandiflora Mart. \& Zucc. No plano de manejo $\mathrm{PMF}_{16}$, na área de manejo florestal, das 109 espécies encontradas, $25(22,93 \%)$ foram consideradas raras e na área de reserva legal, das 96 espécies amostradas, 21 espécies $(21,87 \%)$ foram raras. As espécies raras comuns às duas áreas foram Eriotheca sp., Sapium glandulatum (Vell.) Pax, Sciadodendron excelsum Griseb. e Tibouchina granulosa Cogn.

Quadro 2 - Porcentagem do número de indivíduos, por hectare e por grupos ecológicos, das espécies arbóreas dos $\mathrm{PMF}_{28}$, $\mathrm{PMF}_{29}, \mathrm{PMF}_{30}$ e $\mathrm{PMF}_{16}$, em áreas de manejo florestal e áreas de reserva legal, nos Municípios de Porto Firme e Rio Espera, Minas Gerais, em 2005

Table 2 - Percentage of the number of individuals per hectare and per ecological group, of tree species in the $\mathrm{FMP}_{28}, \mathrm{FMP}_{29}$, $\mathrm{FMP}_{30}$ and $\mathrm{FMP}_{16}$, in forest management areas and legal reserve areas, in the municipalities of Porto Firme and Rio Espera, Minas Gerais State, in 2005

\begin{tabular}{|c|c|c|c|c|c|c|c|c|}
\hline \multirow{4}{*}{ Grupo Ecológico } & \multicolumn{8}{|c|}{ Porcentagem (\%) do Número de Indivíduos por Hectare } \\
\hline & \multicolumn{8}{|c|}{ Condições de Floresta } \\
\hline & \multicolumn{4}{|c|}{ AMF } & \multicolumn{4}{|c|}{ ARL } \\
\hline & $\mathrm{PMF}_{28}$ & $\mathrm{PMF}_{29}$ & $\mathrm{PMF}_{30}$ & $\mathrm{PMF}_{16}$ & $\mathrm{PMF}_{28}$ & $\mathrm{PMF}_{29}$ & $\mathrm{PMF}_{30}$ & $\mathrm{PMF}_{16}$ \\
\hline$\overline{\text { Pioneiras (PI) }}$ & 33,87 & 19,73 & 56,59 & 48,89 & 22,48 & 19,89 & 44,62 & 31,70 \\
\hline Secundárias iniciais (SI) & 40,00 & 66,49 & 24,86 & 38,11 & 55,31 & 67,34 & 32,41 & 43,77 \\
\hline Secundárias tardias (ST) & 26,13 & 13,77 & 18,55 & 13,08 & 22,21 & 12,76 & 22,97 & 24,53 \\
\hline
\end{tabular}

R. Árvore, Viçosa-MG, v.31, n.2, p.247-256, 2007 
Quadro 3 - Porcentagem do número de indivíduos, por hectare e por grupos de uso, das espécies arbóreas dos $\mathrm{PMF}_{28}, \mathrm{PMF}_{29}$, $\mathrm{PMF}_{30} \mathrm{e} \mathrm{PMF}_{16}$, em áreas de manejo florestal e áreas de reserva legal, nos Municípios de Porto Firme e Rio Espera, Minas Gerais, em 2005

Table 3 - Percentage of the number of individuals per hectare and per use group, of the tree species in the $F M P_{28}, F M P_{29}$, $F M P_{30}$ and $F M P_{16}$, in forest management areas and legal reserve areas, in the municipalities of Porto Firme and Rio Espera, Minas Gerais State, in 2005

\begin{tabular}{|c|c|c|c|c|c|c|c|c|}
\hline \multirow{4}{*}{ Grupo de uso } & \multicolumn{8}{|c|}{ Percentagem (\%) do Número de indivíduos por hectare } \\
\hline & \multicolumn{8}{|c|}{ Condições de Floresta } \\
\hline & \multicolumn{4}{|c|}{ AMF } & \multicolumn{4}{|c|}{ ARL } \\
\hline & $\mathrm{PMF}_{28}$ & $\mathrm{PMF}_{29}$ & $\mathrm{PMF}_{30}$ & $\mathrm{PMF}_{16}$ & $\mathrm{PMF}_{28}$ & $\mathrm{PMF}_{29}$ & $\mathrm{PMF}_{30}$ & $\mathrm{PMF}_{16}$ \\
\hline 1-Energia & 56,40 & 42,87 & 73,80 & 46,53 & 60,90 & 48,20 & 62,33 & 56,37 \\
\hline 2-Serraria & 43,60 & 57,13 & 26,20 & 53,47 & 39,10 & 51,80 & 37,67 & 46,63 \\
\hline Total & 100,00 & 100,00 & 100,00 & 100,00 & 100,00 & 100,00 & 100,00 & 100,00 \\
\hline
\end{tabular}

Em média, na área de manejo florestal e na área de reserva legal de todos os $\mathrm{PMF}_{\mathrm{s}}, 24 \%$ do total de espécies amostradas foram consideradas raras, o que concorda com Gomes et al. (2004). Esses autores, estudando dois fragmentos de floresta estacional semidecidual, no Município de Matias Barbosa, decorridos seis anos das atividades de exploração florestal, observaram que a área de manejo florestal apresentou 26,6\% de espécies raras e a área de reserva legal, 36,21\%. Porém, só o monitoramento de longo prazo dessas áreas de florestas irá permitir verificar se as referidas espécies persistem na condição de espécies raras.

O verificador espécies raras mostrou diferenças não-significativas $(\mathrm{P}>0,05)$ entre as áreas de manejo florestal e a área de reserva legal de todos os $\mathrm{PMF}_{\mathrm{s}}$.

\subsection{Diversidade de espécies}

As áreas manejo florestal e as de reserva legal apresentaram alta diversidade de espécies arbóreas (Quadro 4), estimada pelos índices de diversidade de Shannon (H'), equabilidade de Pielou (J'), diversidade ecológica de Simpson (C) e coeficiente de Mistura de Jentsch (QM)

Os valores de H' das áreas de manejo florestal de todos os planos de manejo estão dentro do intervalo 3,2 a 4,2 de valores de H', obtidos para florestas estacionais em Minas Gerais, conforme trabalhos de Oliveira Filho et al. (1994); Meira-Neto et al. (1997); Ameida Jr. (1999); Marangon (2003); Silva et al. (2004).

$\mathrm{Na}$ área de manejo florestal, no referido intervalo se enquadram os valores de H' dos $\mathrm{PMF}_{28}$ e $\mathrm{PMF}_{16}$ (Quadro 4).
Quando foi analisado o intervalo de confiança Jackknife (NETER et al., 1992; HELTSHE e FORRESTER, 1983), verificou-se que os valores de H' das áreas de manejo florestal e áreas de reserva legal eram iguais $(\mathrm{P}>0,05)$ no $\mathrm{PMF}_{28}$ e diferentes $(\mathrm{P} \leq 0,05)$ nos $\mathrm{PMF}_{29}$, $\mathrm{PMF}_{30}$ e $\mathrm{PMF}_{16}$.

O verificador diversidade de espécies $\left(\mathrm{H}^{\prime}\right)$ apresentou (Quadro 5) diferenças significativas $(\mathrm{P} \leq 0,05)$ pelo teste t, de Student, modificado por Magurran (1988), em todos os $\mathrm{PMF}_{\mathrm{s}}$.

\subsection{Similaridade de espécies}

No $\mathrm{PMF}_{28}$, na área de manejo florestal e na área de reserva legal foram amostradas, respectivamente, 83 e 66 espécies, respectivamente, sendo 44 delas $(\mathrm{SO}=59 \%)$ comuns às duas condições de floresta. Dentre as espécies comuns, as de maior VI foram: Maprounea guianensis Aubl., Casearia decandra Jacq., Ocotea sp., Morta, Mabea fistulifera Mart., Tapirira guianensis Aubl., Indeterminada, Lacistema pubescens Mart., Miconia cinnamomifolia Naudin e Siparuna guianensis Aubl. (Figura 3).

No $\mathrm{PMF}_{29}$, na área de manejo florestal e na área de reserva legal foram amostradas, respectivamente, 46 e 59 espécies, sendo $37(\mathrm{SO}=70 \%)$ comuns às duas áreas. Dentre as espécies comuns, as de maior VI foram: Mabea fistulifera, Xylopia brasiliensis Spreng., Rollinia silvatica Mart., Casearia decandra, Lacistema pubescens, Miconia cinnamomifolia, Maprounea guianensis, Morta, Myrcia fallax DC., Erythroxylum sp. e Prunus sellowii Koehne.

R. Árvore, Viçosa-MG, v.31, n.2, p.247-256, 2007 
Quadro 4 - Índices de diversidade florística dos $P M F_{28}, P M F_{29}, P M F_{30}$ e $P M F_{16}$, em áreas de manejo florestal e áreas de reserva legal, nos Municípios de Porto Firme e Rio Espera, Minas Gerais, em 2005

Table 4 - Indexes of floristic diversity of the $F M P_{28}, F M P_{29}, F M P_{30}$ and $F M P_{10}$ in forest management areas and legal reserve areas, in the municipalities of Porto Firme and Rio Espera, Minas Gerais State, in 2005

\begin{tabular}{|c|c|c|c|c|c|c|c|c|}
\hline \multirow[t]{3}{*}{ Parâmetro } & \multicolumn{8}{|c|}{ Condições de Floresta } \\
\hline & \multicolumn{4}{|c|}{ AMF } & \multicolumn{4}{|c|}{ ARL } \\
\hline & $\mathrm{PMF}_{28}$ & $\mathrm{PMF}_{29}$ & $\mathrm{PMF}_{30}$ & $\mathrm{PMF}_{16}$ & $\mathrm{PMF}_{28}$ & $\mathrm{PMF}_{29}$ & $\mathrm{PMF}_{30}$ & $\mathrm{PMF}_{16}$ \\
\hline Coeficiente de Mistura de Jentsch (QM) & 1: 9 & 1: 20 & 1: 10 & 1: 9 & 1: 11 & 1: 16 & 1: 10 & 1: 7 \\
\hline Índice de Diversidade de Shannon (H') & 3,70 & 2,75 & 2,84 & 3,85 & 3,51 & 3,18 & 3,37 & 4,03 \\
\hline Índice de Dominância de Simpson (C) & 0,96 & 0,91 & 0,85 & 0,96 & 0,96 & 0,94 & 0,93 & 0,98 \\
\hline Equabilidade de Pielou (J) & 0,84 & 0,72 & 0,67 & 0,82 & 0,84 & 0,78 & 0,78 & 0,88 \\
\hline
\end{tabular}

Quadro 5 - Índices de diversidade de Shannon (H') e Jacknife, dos $\mathrm{PMF}_{28}, \mathrm{PMF}_{29}, \mathrm{PMF}_{30}$ e $\mathrm{PMF}_{16}$, em áreas de manejo florestal e áreas de reserva legal nos Municípios de Porto Firme e Rio Espera, Minas Gerais, em 2005

Table 5 - Indexes of diversity of Shannon $\left(H^{\prime}\right)$ and Jacknife of the $F M P_{28}, F M P_{29}, F M P_{30}$ and $F M P_{16}$, in forest management areas and legal reserve areas in the municipalities Porto Firme and Rio Espera, Minas Gerais State, in 2005

\begin{tabular}{llcc}
\hline Plano & Parâmetro & AMF & ARL \\
\hline $\mathrm{PMF}_{28}$ & Índice Shannon $\left(\mathrm{H}^{\prime}\right)^{*}$ & 3,7 & 3,51 \\
& Jacnife & 3,57 a 4,10 & 3,43 a 3,77 \\
\hline $\mathrm{PMF}_{29}$ & Índice Shannon $\left(\mathrm{H}^{\prime}\right)^{*}$ & 2,75 & 3,18 \\
& Jacnife & 2,67 a 2,93 & 3,02 a 3,48 \\
\hline $\mathrm{PMF}_{30}$ & Índice Shannon $\left(\mathrm{H}^{\prime}\right)^{*}$ & 2,84 & 3,37 \\
& Jacnife & 2,68 a 3,21 & 3,18 a 3,91 \\
\hline $\mathrm{PMF}_{16}$ & Índice Shannon $\left(\mathrm{H}^{\prime}\right)^{*}$ & 3,85 & 4,03 \\
& Jacnife & 3,85 a 4,13 & 4,01 a 4,3 \\
\hline
\end{tabular}

* Diferença significativa $(\mathrm{P}=0,05)$, pelo teste $\mathrm{t}$, de student, proposto por Hutcheson (1970) e citado por Magurran (1988), entre a área de manejo florestal e a de reserva legal de cada projeto.

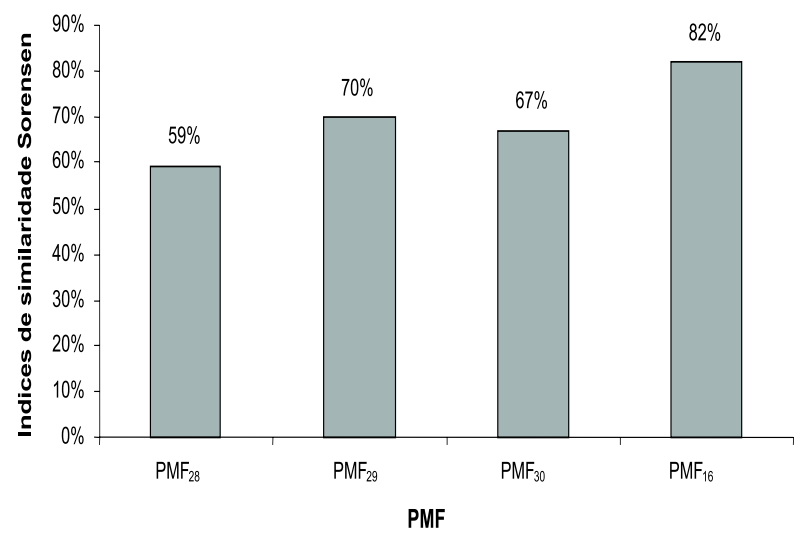

Figura 3 - Índices de Similaridade de Sorensen $(\mathrm{S}), \mathrm{PMF}_{28}$, $\mathrm{PMF}_{29}, \mathrm{PMF}_{30}$ e $\mathrm{PMF}_{16}$, em áreas de manejo florestal e áreas de reserva legal, nos Municípios de Porto Firme e Rio Espera, Minas Gerais, em 2005.

Figure 3 -Indexes of Similarity of Sorensen $(S)$ in the $F M P_{28}$, $F M P_{29}, F M P_{30}$ and $F M P_{16}$, in forest management areas and legal reserve areas in the municipalities of Porto Firme and Rio Espera, Minas Gerais State, in 2005.
No $\mathrm{PMF}_{30}$, na área de manejo florestal e na área de reserva legal foram amostradas, respectivamente, 68 e 75 espécies, sendo $48(\mathrm{SO}=67 \%)$ comuns às duas áreas. Dentre as espécies comuns, as de maior VI foram: Xylopia sericea A.St.-Hill., Apuleia leiocarpa J.F. Macbr., Plahtymenia foliolosa Benth., Pitptocarpha macropoda Baker, Matayba elaeagnoides Radlk., Myrcia fallax, Lacistema pubescens, Siparuna guianensis, Mabea fistulifera, Tabernaemontana fuchsiaefolia Miers e Siparuna sp.

No $\mathrm{PMF}_{16}$, na área de manejo florestal e na área de reserva legal foram amostradas, respectivamente, 106 e 96 espécies, sendo $84(\mathrm{SO}=82 \%)$ comuns às duas áreas. Dentre as espécies comuns, as de maior VI foram: Xylopia brasiliensis, Platypodium elegans Vogel, Pseudopiptadenia contorta (DC.) G.P.Lewis \& M.P.Lima, Anadenanthera peregrina Speg., Dalbergia nigra, Casearia decandra, Mabea fistulifera, Schefflera morototoni (Aubl.) Maguire, Steyerm.\& Frodin, Casearia ulmifolia Cambess., Morta e Trichilia catigua A. Juss. 
O PMF $\mathrm{PM}_{28}$ apresentou o menor índice de similaridade entre as áreas (59\%) e o $\mathrm{PMF}_{16}$, o maior índice (82\%). Apesar da diferença entre riqueza de espécies, o índice de Sorensen revelou alta similaridade florística entre a área de manejo florestal e a de reserva legal de cada PMF. Essa similaridade pode ter ocorrido devido ao fato de as áreas serem muito próximas ou contíguas (Figura 4).

O dendrograma gerado a partir do agrupamento utilizando a distância euclidiana simples e o método de Ward, para os dados de presença e ausência das espécies nas parcelas de cada PMF, permitiu formar, no nível de $90 \%$ de distância euclidiana simples, três grupos distintos de $\mathrm{PMF}_{\mathrm{s}}$ : grupo 1, composto pelos $\mathrm{PMF}_{29 \mathrm{AMF}} ; \mathrm{PMF}_{29 \mathrm{ARL}}$ e $\mathrm{PMF}_{28 \mathrm{ARL}}$; grupo 2, composto pelos $\mathrm{PMF}_{16 \mathrm{ARL}}$ e $\mathrm{PMF}_{16 \mathrm{AMF}}$; e grupo 3, que reúne os $\mathrm{PMF}_{\text {30ARL }}, \mathrm{PMF}_{\text {30AMF }}$ e $\mathrm{PMF}_{28 \mathrm{AMF}}$.

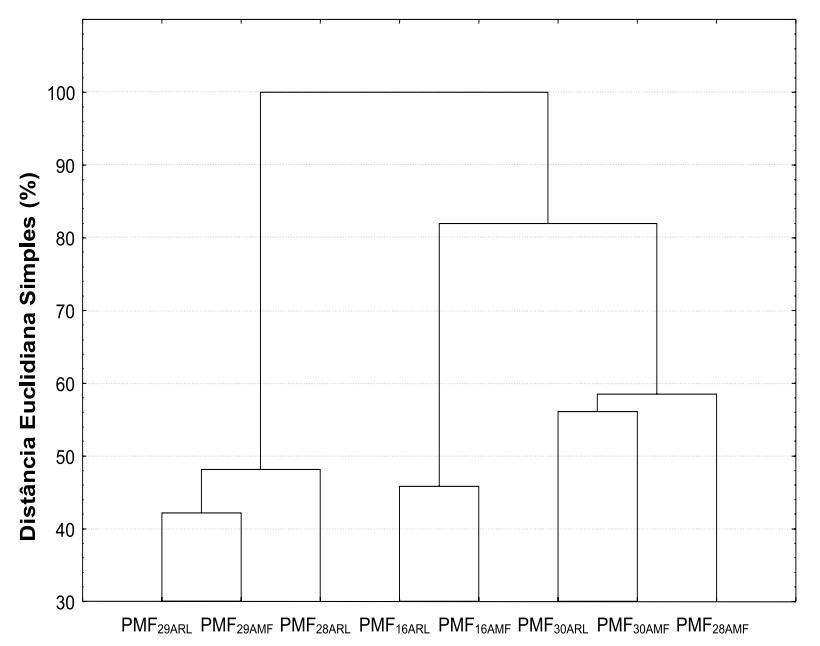

PMF

Figura 4 - Dendrograma obtido da análise de agrupamento, utilizando-se a distância euclidiana simples e o método de Ward, para os dados de presença e ausência das espécies nas parcelas dos $\mathrm{PMF}_{28}, \mathrm{PMF}_{29}, \mathrm{PMF}_{30}$ e $\mathrm{PMF}_{16}$, em áreas de manejo florestal e áreas de reserva legal, nos Municípios de Porto Firme e Rio Espera, Minas Gerais, em 2005.

Figure 4-Dendrogram obtained from the cluster analysis, using the single Euclydean distance and Ward method, for data on presence and absence of species in the plots of the $F M P_{28}, F M P_{29}, F M P_{30}$ and $F M P_{16}$, in forest management areas and legal reserve areas in the municipalities of Porto Firme and Rio Espera, Minas Gerais State, in 2005.

\section{CONCLUSÕES}

Decorrido o tempo de exploração florestal de cada plano de manejo:

- O verificador riqueza de espécies indicou que as áreas de manejo florestal e as de reserva legal dos $\mathrm{PMF}_{28}$ e $\mathrm{PMF}_{29}$ eram estatisticamente iguais, ao passo que nos $\mathrm{PMF}_{30}$ e $\mathrm{PMF}_{16}$, estatisticamente diferentes.

- Os verificadores grupo ecológico, grupo de uso e grupo de espécies raras indicaram que as áreas de manejo florestal de todos os $\mathrm{PMF}_{\mathrm{S}}\left(\mathrm{PMF}_{28}, \mathrm{PMF}_{29}, \mathrm{PMF}_{30}\right.$ e $\left.\mathrm{PMF}_{16}\right)$ eram estatisticamente iguais às áreas de reserva legal.

- O verificador diversidade de espécies indicou que as áreas de manejo florestal são estatisticamente diferentes das áreas de reserva legal nos $\mathrm{PMF}_{29}, \mathrm{PMF}_{30}$ e $\mathrm{PMF}_{16}$ e estatisticamente iguais no $\mathrm{PMF}_{28}$.

- A maior similaridade de espécies ocorreu entre os agrupamentos formados pelos $\mathrm{PMF}_{29 \mathrm{AMF}}$ e $\mathrm{PMF}_{29 \mathrm{ARL}}$ e a menor entre os agrupamentos formados pelos $\mathrm{PMF}_{29 \mathrm{ARL}}$, $\mathrm{PMF}_{29 \mathrm{AMF}}, \mathrm{PMF}_{28 \mathrm{ARL}}$ e $\mathrm{PMF}_{16 \mathrm{ARL}}, \mathrm{PMF}_{16 \mathrm{AMF}}, \mathrm{PMF}_{30 \mathrm{ARL}}$, $\mathrm{PMF}_{30 \mathrm{AMF}}$ e $\mathrm{PMF}_{28 \mathrm{AMF}}$.

\section{REFERÊNCIAS}

\section{ASSEMBLÉIA LEGISLATIVA DO ESTADO DE MINAS GERAIS - ALMG caracterização dos municípios mineiros com base em informações do IGA-CETEC e IBGE. Disponível em: <www.almg.gov.br>.} Acesso em: 7 dez. 2005.

\section{CIENTEC . Mata Nativa 2,}

versão 2: sistema para análise fitossociológica e elaboração de inventários e planos de manejo de florestas nativas. Viçosa, MG, Cientec -

Consultoria e Desenvolvimento de Sistemas, 2006.

DENSLOW, J. S. Gap partitioning among tropical rain forest trees. Biotropica, v.12, p.47-55, 1980.

DENSLOW, J. S. Tropical rain forest gaps and the two major groups of forest trees. Ecology, v.70, p.536-538, 1987.

GOMES, A. P. C.; SOUZA, A. L.; MEIRA NETO, J. A. A. Alteração estrutural de uma área florestal explorada convencionalmente na bacia do Paraíba do Sul, Minas Gerais, nos domínios de Floresta Atlântica. Revista Árvore, v.28, n.3, p.407-417, 2004.

R. Árvore, Viçosa-MG, v.31, n.2, p.247-256, 2007 
HELTSHE, J. F.; FORRESTER, N. E. Estimating species richness using the jackknife procedure. Biometrics, v.39, p.1-11, 1983.

INSTITUTO ESTADUAL DE FLORESTAS - IEF. Contrato BIRD/ SEPLAN/IEF-MG. Programa Prófloresta. Cobertura vegetal e uso do solo do Estado de Minas Gerais. Belo Horizonte: 1994. 1 mapa: color. 99x66. Escala: 1:1.500.000. Material cartográfico.

INSTITUTO ESTADUAL DE FLORESTAS - IEF. Banco de dados sobre planos de manejo florestal em Minas Gerais. Viçosa-,MG: 2000. 10p.

JARDIM, F. S. C.; VOLPATO, M. M. L.; SOUZA, A. L. Dinâmica de sucessão natural em clareiras de florestas tropicais. Viçosa, MG: SIF, 1993. 60p.

LILLIEFORS, H. W. One The kolmogorov-smirnov test for normality with mean and variance unknown. Jornal of the American Statistical Association, v.64, p.399-402, 1967.

LORENZI,.H. Árvores brasileiras: manual de identificação e cultivo de plantas arbóreas nativas do Brasil - Nova Odessa-SP: Editora Plantarium, 1992.368p.

\section{MAGURRAN, A. E. Ecological diversity}

and its measurement. Princeton: Princeton University Press, 1988. 167p.

MARAGON, L. C. Florística arbórea da mata da pedreira, município de Viçosa, Minas Gerais. Revista Àrvore, v.27, n.2, p.207-215, 2003.

MEIRA NETO, J. A. A. Estudos florísticos, estruturais e ambientais nos estratos arbóreo-arbustivos de uma floresta estacional semidecidual em Viçosa, MG. 1997. 154f. Tese (Doutorado em Ciências Biológicas) Universidade Estadual de Campinas, Campinas, 1997.
NETER, J.; WASSERMAN, W.; WHITMORE, G. A. Applied statistics. Englewood Cliffs: Prentice Hall, 1992. p. 463-466.

OLIVEIRA FILHO, A. T.; SCOLFORO, J. R. S.; MELLO, J. M. Composição florística e estrutura comunitária de um remanescente de floresta semidecídua montana em Lavras, MG. Revista Brasileira de Botânica, v. 17, n.2, p.167-182, 1994.

SILVA, R. S. S. et al. Composição florística e estrutura de uma floresta estacional semidecidual montana em Viçosa, MG. Revista Árvore, v.28, n.3, p.397-405, 2004.

FUNDAÇÃO ARTHUR BERNARDES -FUNARBE. Sistema para análises estatísticas SAEG, versão 9.0. Viçosa, MG: 2005.

VELOSO, H. P. et al. Manual técnico da vegetação brasileira. Rio de Janeiro: IBGE, 1992. 92 p. (Série Manuais Técnicos em Geociências, 1).

VIDAL, E.; VIANA, V.; BATISTA, J. L. F. Efeitos da exploração madeireira predatória e planejada sobre a diversidade de espécies na Amazônia Oriental. Revista Árvore, v.22, n.4, p.503-520, 1998.

WHITMORE, T. C. Canopy gaps and the two major groups of forest trees. Ecology, v.70, n.3, p.536-538, 1989.

WHITMORE, T. C. Gaps in the forest canopy. Tropical trees as living systems. London: Cambridge University, 1978. p.536-538.

WINER, B. J. Statistical principles in experimental design. New york: Megrawwill, 1962. 94p. 